\title{
A Tentative Exploration of Structuring Information in Community Emergency Management Risk Radar by Presetting Forms
}

\author{
Yifang Leng ${ }^{1,2,3}$, Chongfu Huang ${ }^{1,2,3, *}$ \\ ${ }^{1}$ Key Laboratory of Environmental Change and Natural Disaster, Ministry of Education of China, Beijing \\ Normal University, Beijing 100875, China \\ ${ }^{2}$ State Key Laboratory of Earth Surface Processes and Resource Ecology (Beijing Normal University), Beijing \\ 100875, China \\ ${ }^{3}$ Academy of Disaster Reduction and Emergency Management, Ministry of Civil Affairs \& Ministry of Education, the \\ Peoples' Republic of China, Beijing 100875, China
}

\section{社区应急管理风险雷达中预设表单式信息结构化研究初探 \\ 冷一芳 ${ }^{1,2,3}$, 黄崇福 $1,2,3$, * \\ ${ }^{1}$ 北京师范大学环境演变与自然灾害教育部重点实验室, 北京 100875 , 中国 \\ ${ }^{2}$ 地表过程与资源生态国家重点实验室(北京师范大学), 北京 100875, 中国 \\ ${ }^{3}$ 民政部/教育部减灾与应急管理研究院, 北京 100875 , 中国}

\begin{abstract}
this paper proposed a method to help transform the unstructured data of community emergency management risk radar, by understanding the risk awareness and expression habit of community residents, a series of forms can be developed to summarize the problem, them the Multiple Internet of Intelligences can pop up the most appropriate form to get the structured information, in this way, the community emergency management risk radar can continuous work, and we can achieve the goal of community disaster reduction. Keywords: community, emergency management, risk radar, internet of intelligences, structuring information

\section{摘要}

本文为解决社区应急风险雷达中非结构化数据转化 问题, 提出以智联网驱动的社区风险雷达为研究对

基金项目：国家自然科学基金项目（编号：41471424）, 国家重大科学研究计划 “全球变化与环境风险演变过程与 综合评估模型” (编号：2012CB955402)。

*通讯作者: 黄崇福 (1958-), 男, 云南人, 博士, 教授, 主要研究方向: 风险分析, 智联网, E-mail: hchongfu@bnu.edu.cn
\end{abstract}

象, 通过深入了解社区居民的风险意识和表述习惯, 研制一系列总结过程中使用的表单, 使复式智联网能 根据获得的风险问题弹出最佳的表单并获取相应的 结构化经验信息, 形成自动运行的复式智联网, 持续 驱动社区风险雷达, 达到社区减灾的目的。

关键词: 社区, 应急管理, 风险雷达, 智联网, 信息 结构化

1. 引言

在高风险社会, 人口密集的社区一旦发生灾害, 损失往往十分巨大, 如天津港 “ 8.12 ” 爆炸, 造成巨 大伤亡。社区是社会的基本单位, 也是城市管理的基 本单元。从二十世纪末开始, 社区减灾就已经是国内 外采用的减灾战略之一。1999 年联合国在 “国际 防灾战略” (ISDR) 中提出 “ 21 世纪建设更安全的 世界” 的三大战略之一就是 “从强调政府的作用到重 视推进建设灾害应对能力强的社区” ${ }^{[1]}, 2001$ 年国 际减灾日的行动口号是“发展以社区为核心的减灾战 略”, 2005 年联合国减灾大会也明确提出, “在所有 的社区阶层, 特别是在社区建立防灾机制和提高防灾 能力”。我国《国家综合减灾 “十一五” 规划》中也 将创建综合减灾示范社区作为国家减灾的重要内容 [2]］社区灾害风险管理是治理和减少风险并确保可持 
续发展的有效手段 ${ }^{[3]}$, 在社区减灾管理中, 如果社区 居民可以将已有的经验贡献出来, 也可以为社区减灾 奉献自己的力量。

国外关于社区减灾领域的研究整体上起步较早, 在公众参与社区减灾管理的应用系统和相关技术方 面的研究主要集中在人机交互系统以及社区灾害信 息系统的设计和应用等方面。Steve Carver 等人指 出互联网、在线空间决策支持系统如地理信息系统为 公众参与提供了人-机协作的技术平台 ${ }^{[4]}$, Alan MacEachren 等人通过分析危机情景下基于地理信息 的个人和团队工作中的信息问题, 应用协同同一地点 和不同地点的危机管理活动的地理信息技术, 设计开 发了计算机支持的协同工作系统 ${ }^{[5]}$, Dransch D. 基 于风险沟通的特殊障碍和挑战, 提出了公众交互性风 险地图的设计思路以及应用框架 ${ }^{[6]}$ 等。

国内也已经有许多包括安全社区建设、社区防灾 减灾、社区安全文化建设等方面的研究。如沙勇忠等 介绍了美国减灾型社区建设以及给我们的启示 ${ }^{[7]}$ 。伍 国春介绍了日本 “灾害文化” 的建设以及日本社区防 灾减灾体制与应急能力建设模式 ${ }^{[8]}$ 。郭瑞参考了联合 国环境署的本地化应急响应方案的做法, 研究了构建 我国 “奥运安全社区” 的建设框架和运行模式 ${ }^{[9]}$ 。 杨洋、叶韵在对我国构建安全社区的现实性进行分析 的基础上, 提出了构建城市安全社区的十个步骤 ${ }^{[10]}$, 刘刚以兰州市某社区为案例, 进行协作式风险信息地 图系统的建立进行社区危机灾害管理 ${ }^{[11]}$ 。这些研究主 要是从宏观上探讨公众的参与机制与协作, 对公众参 与社区减灾技术进行具体研究较少。

在 “人人都是传感器” 的互联网时代, 人机结合 的智联网, 正在推动风险分析进入互联网的大数据时 代。智联网驱动的, 用于隐患排查的初级风险雷达, 已经能为社区居民参与风险防范与风险治理提供有 效途径。然而, 目前的风险雷达, 在复式智联网处理 社区居民提供的信息时, 在交叉验证和综合提取, 实 现非结构化信息向结构化信息的转化环节中, 所使用 的表单, 是由操作人员现用现制, 形成信息流中的肠 梗阻, 制约了风险雷达的发展。

\section{2. 智联网简介}

智联网 (Internet of Intelligences) 是由各 种智能体, 通过互联网形成的一个巨大网络。其目的 是集小智慧为大智慧, 群策群力, 帮助人们更好地认 识世界, 获得更好的生活质量。根据黄崇福 ${ }^{[12]}$ 的观点, 智联网是由智能体、网络、模型组合而成的。智联网 的定义是, 设 $\mathrm{A}=\{\mathrm{a} 1, \mathrm{a} 2, \cdots \cdots$, an $\}$ 是含有 $\mathrm{n}$ 个智能
体的集合, $\mathrm{N}=\{\mathrm{S}, \mathrm{c} 1, \mathrm{c} 2, \cdots \cdots, \mathrm{cn}\}$ 是含有服务器 $\mathrm{S}$ 和 $\mathrm{n}$ 台计算机网络终端 $\mathrm{c} 1, \mathrm{c} 2, \cdots \cdots, \mathrm{cn}$ 的一个可以 独立工作的子系统, $\mathrm{M}$ 是处理 $\mathrm{n}$ 个智能体提供的信息 的模型, 三元体 $\langle A, N, M>$ 称为一个智联网, 如图 1 所示。

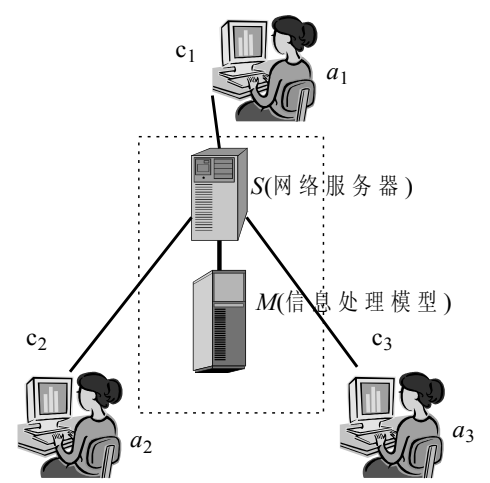

图 1. 最简单的智联网

在灾害学领域, 虽然国内已有各种关于灾害的研 究, 但许多承灾体的破坏机理还处于假说阶段或是并 未完全研究清楚, 同时, 承灾体的关键数据获取困难, 精度不够, 以此为基础进行的脆弱性分析是不确定 的, 在输入-输出关系上, 只能表达为多值对应的关 系, 而非一对一的输入-输出关系。为表达这种不确 定的关系, 黄崇福教授提出可以用模糊关系表达, 也 就是信息扩散 ${ }^{[13]}$ 。而智联网的核心正在于模型的独特 性, 它采用了以信息扩散为基础的理论模型, 使得它 可以集众人之智。

智联网通过网络的获得利益相关者和专家等智 能体提供的风险信息, 主要为柔性信息 ${ }^{[14]}$ 。柔性地理 信息往往包括两个层次的内容, 一是与地理有关的空 间特征部分, 可以是有经纬度坐标组成的点、线、面 等, 或者由地名, 街道名、地标兴趣点等描述性文字 表示的地理位置; 二是属性特征部分, 可以包括与目 标地物相关的任意方面的信息。在处理和分析这类信 息时, 应用复式智联网模式, 让提供经验的智能体再 次参与。复式智联网中, 智能体 $\mathrm{A}$ 和 模型 $\mathrm{M}$ 的智能 反映了智联网的智能, 当参与智联网服务的智能体 $\mathrm{A}$ 通过网络 $N$ 成为信息处理模型 $M$ 的一部分时, 智能 体 A 既是智慧服务的信息提供者又是信息分析处理 的参与者, 可以对其他智能体提供的经验信息进行评 估, 而利用人脑知识库及人脑的智能作用对经验信息 进行处理与分析, 也是风险分析模型的一部分 ${ }^{[15]}$ 。 


\section{Risk Analysis and Crisis Response in Big Data Era (RAC-16)}

\section{3. 社区应急管理风险雷达}

雷达, 是指一种发射和接受电磁波用以对目标物 进行监测从而获得其位置及移动等信息的电子设备, 而发现、识别、监测和管理动态风险的系统，称为风 险雷达。在雷达机制原理的模仿启发下, 1996 年, 人们首次提出风险雷达的概念。 2005 年, 新加坡政 府提出用 “风险雷达” 监测恐怖主义活动及其他国内 的安全威胁事件, 该系统拟通过收集和智能重组相关 数据来帮助政府部门机构利用这些有效的风险信息 完善国家安全工作。欧盟第七框架计划大型项目 iNTeg-Risk 提出的欧洲新兴风险雷达, 则是用以监 测今后情景, 包括对于新兴风险事件的辨识和追踪, 为科研界和工商业和公众提供大量有价值可以信赖 的风险信息 ${ }^{[16]}$ 。欧洲新兴风险雷达, 由一系列同心圆 和辐射线组成, 见图 $2^{[17]}$ 。Jovanovic 和他的研究团 队研究中的欧洲风险雷达, 主要是利用 twitter 等社 交媒体来追踪人们的关注热点, 并以此获取经济、环 境、政治和科技方面的风险相关信息。

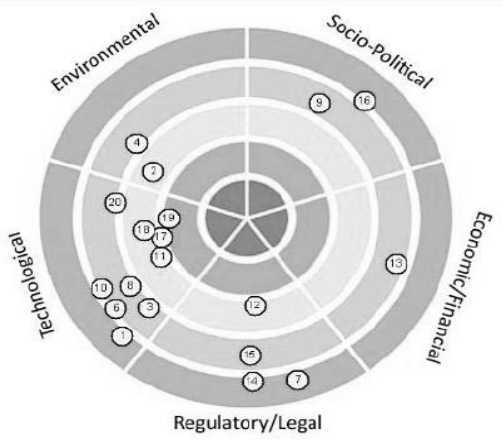

图 2. 建设中的欧洲新兴风险雷达示意图

在国内, 风险雷达的概念主要运用在與情监测、 企业风险监测和社区应急监测中。商业银行的风险监 测中 “银行风险雷达图” 是将银行的财务状况及经 营成果等信息用各类比率的形式在雷达网状图上显 示出来, 以便监管人员了解银行运作的风险状况 ${ }^{[18]}$ 然而, 除了以雷达图的形式来表达风险信息, 风险雷 达还应当是风险评估和管理、处理不同数据一个信息 系统, 是一种从多方面进行风险事件相关数据的收集 和智能组织处理用以帮助政府机构防范安全威胁, 为 公司企业和公众提供有效可信的信息, 帮助其合理规 避不理风险事件, 进行风险因子预警显示的风险管理 工具系统 ${ }^{[19]}$ 。
我国是第一个尝试将风险雷达用于提高社区应 急管理能力的国家, 使用智联网驱动技术。与传统商 用风险雷达不同的是, 智联网驱动的风险雷达是一个 在线系统, 而不再是传统风险矩阵的一种雷达化显示 ${ }^{[17]}$; 与 Jovanovic 式欧洲风险雷达不同的是, 智联网 驱动的风险雷达是一个人机交互系统, 而不是对网页 海量信息进行归纳总结的系统。智联网驱动的风险雷 达, 针对性更强, 提供的风险预警信息更精细。

智联网驱动风险雷达的基本原理是: 社区居民用 手机或台式电脑, 通过智联网平台, 提供其所发现的 社区隐患信息和风险判断。经交叉验证和综合提取, 实现非结构化信息向结构化信息的转化。网络服务器 中的风险分析模型对这些信息进行处理, 形成以风险 分类和风险水平为指标的风险评估结果, 经由互联网 推送到手机和台式电脑的风险雷达图上, 达到监测和 跟踪风险的目的, 为社区应急管理提供风险预警服 务。基本原理图见图 3。

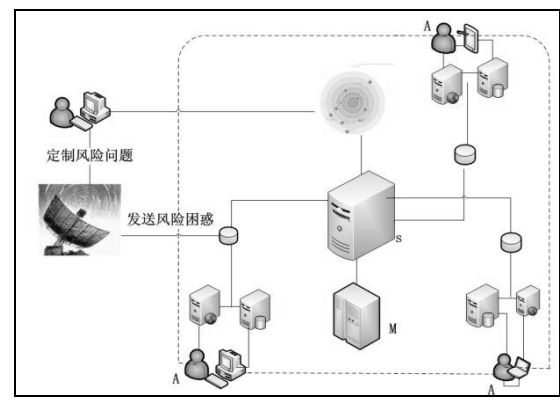

图 3. 智联网驱动的风险雷达原理示意图

在社区减灾中，社区居民有丰富的应对灾害风 险的经验, 并能感知未来的风险, 让居民参与到社 区减灾中, 有利于发挥公众及其他组织在防灾减灾 中的作用 ${ }^{[20]}$ 。将风险雷达的理念和社区减灾结合在 一起, 就形成了一种收集与监测社区潜在风险, 借 助风险雷达动态展示社区风险变化, 为社区管理提 供风险监测与预警的管理系统, 即社区应急管理风 险雷达。目前已开发的风险雷达包括手机客户端应 用软件与网页平台，通过社区居民与社区工作人员 共同参与风险管理方式，对社区风险事件进行信息 采集, 风险分析和反馈展示, 实现社区风险的动态 展示与跟踪 ${ }^{[21]}$

目前, 阻碍智联网有效驱动风险雷达一个主要 问题是智联网获得的风险信息大多是非结构化信 息。结构化数据是可以存储在数据库里, 用二维表 的逻辑结构来表达的数据, 而除此之外, 数据结构 
不固定, 无法使用关系数据库存储, 只能够以各种 类型的文件形式存放的文档、文本文件、图片、图 像、音频和视频等称为非结构化数据 ${ }^{[2]}$ 。智能体提 供的经验信息是人类感知风险的自然语言表述, 是 非结构化数据, 不能简单地规范化和数量化, 如自 然灾害中的 “洪水齐腰深”、“漂泼大雨” 等, 这类 信息在经验信息中占有较大比例, 而大多数分析模 型能够处理的数据是以规范的和量化的形式表示的 ${ }^{[15], ~}$ 因而社区风险雷达的运行还离不开人工的处理 环节。

知名调研机构 Forrest Research 的统计资料表 明, 只有 $15 \%$ 左右的信息有效存储在了各种类型的 结构化数据库中, 还有约 $85 \%$ 的非结构化信息分散 存放的, 其中只有不到 $5 \%$ 的数据进行了数字化管理

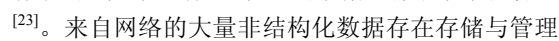
方面的问题, 而解决这一问题目前通常的思路是是 通过 “非结构化数据一半结构化数据一结构化数据” 的方式实现数据内容从非结构化向结构化的转换, 从而对非结构化数据进行统一有效的管理 ${ }^{[24]}$, 但相 关研究大多只是针对于某一类型的文件展开的, 而 目前研制的社区风险雷达 ${ }^{[19]}$ 在社区运行过程中搜集 的数据类型包括数字、图片、描述性的大段文字等, 当中的非结构化信息必须在人工进行处理之后才能 进入模型进行计算。当风险雷达在更多社区中运用 时, 接收到的大量风险信息显然不能再依靠人工处 理, 因此, 如何将非结构化数据转为结构化数据, 也是风险雷达面临的几大挑战之一。

随着《中共中央国务院关于进一步加强城市规 划建设管理工作的若干意见》的印发, 我国新建住 宅要推广街区制, 原则上不再建设封闭住宅小区, 同时逐步打开封闭式小区, 由此带来了道路利用率 提高等便利, 但却为社区安全带来了更大的隐患。 在此背景下, 推广社区应急管理风险雷达, 对提高 我国社区安全具有重要意义。但是, 目前的社区应 急管理风险雷达还在初级建设阶段, 一些环节中的 获得的非结构化信息的数据处理仍然依靠人工操 作, 尚无法应对大面积使用带来的大量数据, 不能 达到自行运行的目标。

\section{4. 预设表单式社区应急管理风险雷达}

为解决社区应急风险雷达中信息处理问题, 可 以通过深入社区调研，对社区各种资料进行搜集整 理, 同时研究和总结各种社区灾害事件, 提取面向 各类风险问题所需的数据, 研制一系列总结过程中 使用的表单, 使复式智联网能根据获得的风险问题
调出该风险问题对应的预设表单, 获取相应结构化 的经验信息, 形成自动运行的复式智联网, 持续驱 动社区风险雷达。本研究是一项试验性研究, 先选 择几个社区进行初步分析, 再根据反馈进一步设计 和完善复式智联网网络平台的功能。

\section{1. 社区调研}

通过深入社区对社区居民和社区工作人员进行 问卷调查和访谈, 进一步了解和完善社区的风险类 型、灾害事件以及居民的风险意识和表述习惯, 对 社区的地理环境、居民分布、建筑情况、历年自然 灾害、人为灾害和社区灾害事件处理经验等情况做 进一步收集整理, 在全面把握社区减灾状况的基础 上对各种社区灾害事件进行研究和总结, 提取面向 各类风险问题所需的数据。

\section{2. 数据结构化转化和表单设计}

社区风险雷达搜集到的信息包括以数字形式表 示的危害度、紧急度和事件类型等信息, 同时还包 括大段文字的详情描述以及辅助描述的图片等信 息, 后者属于非结构化信息, 虽然可利用复式智联 网中人脑的智能作用参与对经验信息进行处理与分 析 $^{[15]}$, 但效率较慢, 也不方便连接到模型进行运算。

为解决风险雷达中非结构化信息转化问题, 在 风险雷达开发阶段, 通过预设信息搜集页面的表单, 利用智能体的人工智能将复杂的详情描述文字信息 转化为简单的选择填空表单形式, 以此搜集各类风 险问题所需的数据。以社区积水问题为例 ${ }^{[15]}$, 设计 者将道路分解为若干路段, 在此基础上搜集到的经 验信息见表 1 , 其中的详细经验信息是非结构化的文 本, 只能依靠人工提取, 费事费力。

为实现积水问题详情经验信息的数据结构化, 在对社区积水事件进行整体研究之后, 可以提取出 积水问题所需数据, 并设计好对应的表单, 初步设 计为, 搜集包括积水路段, 积水开始时间、持续时 间、积水水质状态、积水深度、积水位置、个人灾 害损失、设备破坏情况等数据, 并对每个信息的数 据结构进行界定, 如积水深度设置为整型, 并给定 单位。在风险雷达信息采集页面设计以填空或选择 的方式提交的表单, 此外, 为减少预设表单提取信 息方式带来的柔性信息丢失的问题, 还提供可以补 充信息的文本框, 更大限度地搜集相关信息。同时, 系统也会参考智能体对问题的补充, 不断完善表单。 简单表示如图 4, 通过预设表单的形式利用人脑的思 维将非结构化信息转化为可以直接存储和运算的信 息。 
Risk Analysis and Crisis Response in Big Data Era (RAC-16)

表 1 积水问题经验信息表

\begin{tabular}{ll}
\hline 信息来源 & 详细经验信息 \\
\hline $\mathrm{A}$ & 幼儿园至西门路段积水较深, 据说 \\
& 最深可达膝盖部位, 目测大约 \\
& $40 \mathrm{~cm}$ 左右 \\
& 化学楼和艺术楼之间的路上会积 \\
& 水 \\
& 从地遥楼到学 16 楼, 途中地遥楼 \\
& 门口有积水, 目测深度在 3 厘米 \\
& 左右 \\
& 23 积水 $30 \mathrm{~cm}$, 汇流, 在两边的人 \\
& 行道走, 水多看不清陷入路边树木 \\
& 倒倒后 形成的根部泥坑, 拔出凉 \\
& 鞋, 后部鞋带子撕裂 \\
\hline
\end{tabular}

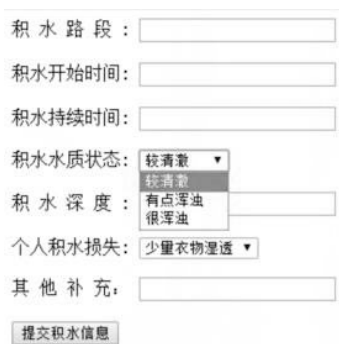

图 4 填空或选择的方式提交表单

\section{3. 平台搭建}

结合以往智联网开发的经验与实际需求, 选择 WAMP 软件包（Windows + Apache+Mysql+PHP）， 一款由法国人开发的 Apache Web 服务器、PHP 解释 器以及 MySQL 数据库的整合软件包) 为平台开发环 境, YII 框架为平台主框架。具体为, 使用 PHP （Hypertext Preprocessor, 超文本预处理语言）, 一 种在服务器端执行的嵌入 HTML 文档的脚本语言来 创建动态交互性站点, 更快速地执行动态网页; 使 用关系型数据库管理系统 MYSQL 来存储数据; 使 用基于组件、用于开发大型 Web 应用的高性能 PHP 框架 Yii 框架来搭建智联网网站。

首先以本地机器为服务器, 安装 WAMP 服务 环境, 搭建 YII 框架, 检测系统开发环境, 建立 PHP 工程。主工程搭建成功后, 连接 MYSQL 数据库, 对网页进行改造和设计, 使得主界面显示包含各功
能模块的菜单等。在生成的框架基础上添加智联网 的核心功能模块, 程序全部开发完成后, 可在真实 的网络环境中发布并进行应用 ${ }^{[15]}$ 。

功能上智联网将包括确定和发布问题, 经验信 息采集, 风险评估, 动态风险表达和总结报告四个 部分。

\section{(1) 确定和发布问题}

对人类而言, 碰到的困扰对个体而言是独一无 二的, 但又是很多人曾经遇到过的, 因此, 他人的 经验往往可以帮助解决个体的特殊困惑, 这也是百 度知道等在线问答平台的创设原理。而智联网与百 度知道相比, 它能用某些数学模型处理来自不同智 能体的信息, 给出总结性的解答, 而不是社交网络 上的离散回答 ${ }^{[17]}$ 。一般地, 智联网是利用需求定制 器来完成问题的和采集和确定。此阶段, 需求者可 以登录智联网平台, 提出自己的困惑或发现的问题, 等待其他智能体参与并解答问题。问题可以分为两 类, 一类属于普通问题, 可以通过简单的表单发布 到智联网平台, 以获得较高效率的解决。另一类问 题则比较复杂, 在智联网研制阶段, 需要研究者搜 集资料, 在对问题进行深入研究之后设计对应表单, 发布在平台上, 以供智能体提供更为丰富的经验信 息。

(2) 经验信息采集

智联网平台发布问题之后, 智能体通过登陆智 联网进入相应问题页面给予回答, 对于普通问题, 如居民举报违规用电现象, 在吴娅的风险雷达中 ${ }^{[19]}$, 系统采取了对危险和紧急程度打分制, 加以详细描 述的方式来处理的。本研究中, 对于普通问题, 可 能沿用打分制加详情描述、建议处理方式等搜集经 验信息, 同时将添加提交类似情况的模块, 如社区 内有人举报有可疑人员在某居民楼前停留并做标 记, 智能体通过智联网回答该问题, 并扩大和补充 可疑人员在社区的活动范围, 为社区管理人员更完 善地解决隐患提供情报。对于复杂问题, 则通过预 设表单搜集智能体的经验信息。对于预设模板之外 的复杂问题, 可以提供一个讨论模块, 智能体畅所 欲言, 充分发掘问题的方方面面, 供后台参考并完 善问题表单模板。

(3) 风险评估

智能体提交经验信息后, 智联网会将这些信息 发布在网上, 接下来进行二次评估阶段, 利用人脑 的智能作用参与对经验信息进行初步处理与分析。 复式智联网中经验信息提供者将根据自身经验对其 他智能体的经验信息进行提取、评判与打分, 通过 
二次评估和交叉提取, 对该经验信息进行判定, 通 过设定阈值, 排查出恶意信息和虚假信息, 以免干 扰之后的模型处理。

在得到二次参与和判定之后的经验信息集的表 单之后, 本研究采用包括信息扩散在内的数学模型 进行风险评估, 其中, 风险信息扩散技术是用来弥 补小样本的不完备缺憾, 将普通样本转化为模糊样 本, 提高结果的客观性。

扩散方法定义如下:

令 $X=\left\{x_{1}, x_{2}, \ldots, x_{i}, \ldots, x_{n}\right\}$ 为区间 $[0,1]$ 的随机 样本, 利用正态扩散函数将 $\mathrm{xi}$ 变为模糊集 ${ }^{[12]}$,

$$
\begin{aligned}
& \left.\mu_{x_{i}}=\exp \left(-\frac{(x-u)^{2}}{2 h^{2}}\right), u \in[0,1] \quad \text { ( } 1\right) \\
& f(u)=\sum_{i=1}^{n} \mu_{x_{i}}(u), u \in[0,1] \\
& f\left(u_{0}\right)=\sup \sup _{\mathrm{u} \in[0,1]} f(u), \\
& \operatorname{diffusing}\left(x_{1}, x_{2}, \ldots, x_{i}, \ldots, x_{n}\right)=u_{0(}(4)
\end{aligned}
$$

除信息扩散模型外，还将基于实验风险学的思 想, 利用智联网平台对不同的数据模型进行测试, 从而对参与实验的模型进行优劣排序, 选择出最佳 模型 ${ }^{[25]}$ 。

\section{（4）动态风险表达和总结报告}

风险时时刻刻都在变化, 尤其社区事件发生快, 变化明显, 风险的动态性更是不可被忽略。随着时 间的推移，不断有智能体加入到风险评估中，提供 自己的经验信息, 此时发生变化的不仅仅是风险评 估的结果, 还包括风险事件本身。因此, 实时的风 险更新是不可或缺, 为了更好的反映事件的变化, 评估结果是实时更新的。

社区风险雷达以智联网为驱动内涵, 获得不断更 新的风险评估结果, 并在风险雷达图中表现为事件 状态的不断更新。此外, 为达到更好的社区减灾目 的, 智联网平台可以生成事件总结报告, 并提供下 载的功能, 为需求者和社区工作人员提供风险事件 的完整信息, 同时还可以给其他智能体提供更为丰 富的经验信息，减少类似问题的重复发生。

\section{5. 结论和讨论}

目前, 社区应急风险雷达通过智联网获得的经验 信息大多是非结构化信息, 是人类感知风险的自然
语言表述，而大多数分析模型能够处理的数据是以 规范的和量化的形式表示的，因而社区风险雷达运 行还离不开人工的处理环节。

本文初步提出了社区应急风险雷达中信息结构 化研究的框架, 针对智联网驱动的社区应急管理风 险雷达中一些环节的数据处理不能在线实现, 从而 不能持续驱动风险雷达的问题, 本研究拟通过深入 社区调研, 对社区各种资料进行搜集整理和深入研 究, 提取面向各类风险问题所需的数据, 研制一系 列总结过程中使用的表单，使复式智联网能根据获 得的风险问题调出该风险问题对应的预设表单，获 取相应结构化的经验信息, 形成自动运行的复式智 联网, 持续驱动社区风险雷达。

\section{参考文献}

[1] 陈徐东. 社区防灾减灾保障机制的创新. 城市与 减灾, 2010(6):5-7.

[2] 卫敏丽. 解读《国家综合减灾 “十一五” 规划》。 Disaster Reduction in China, 2007(9):19-22.

[3] 陈容,崔鹏.社区灾害风险管理现状与展望.灾害 学, 2013, 28 (1) : 133-137.

[4] S. Carver. Public participation, GIS, and cyberdemocracy: evaluating on-line spatial decision support systems. Environment and Planning B-Planning \& Design, 2001, 28(6): 907-921.

[5] A.M. Maceachren, G.Cai, M. Mcneese. Geocollaborative Crisis Management: Designing Technologies to Meet Real-World Needs, in Proceedings. 7th Annual National Conference on Digital Government Research, San Diego, Ca, 2005.

[6] D. Dransch, H. Rotzoll, K. Poser. The contribution of maps to the challenges of risk communication to the public. International Journal of Digital Earth, 2010, 3(3): 292-311

[7] 沙勇忠, 刘海娟. 美国减灾型社区建设及对我国 应急管理的启示. 兰州大学学报, 2010(02):72-79.

[8] 伍国春. 日本社区防灾减灾体制与应急能力建设 模式. 城市与减灾, 2010(02): 16-20.

[9] 郭瑞. 奧运安全社区与本地化方案研究. 北京, 北京化工大学, 2006

[10] 杨洋, 叶韵. 简论我国城市安全社区的构建. 经 营管理者, 2010, 26(15): 180-184 
Risk Analysis and Crisis Response in Big Data Era (RAC-16)

[11] 刘刚.社区灾害风险的评价模型构建及其应用研 究.兰州大学, 2012

[12] 黄崇福. 自然灾害风险分析与管理. 科学出版 社, 2012

[13] 黄崇福. 自然灾害风险评价理论与实践. 科学 出版社发行处出版社, 2006.

[14] 王蔚丹, 黄崇福, 艾福利. 基于智联网的地震预 报策略初探. 风险分析和危机反应的创新理论 和方法, 黄崇福, 翟国方编. 巴黎: Atlantis 出版 社, pp.50-55, 2012.

[15]艾福利. 自然灾害风险分析智联网服务平台构建 与应用研究.北京: 北京师范大学, 2013.

[16] A. Jovanovic, D. Balos, Y. Liu. The European Emerging Risk Radar Initiative - a Chance for China? Procedia Engineering, 2012, (43): 489 $-493$

[17] 黄崇福.自然灾害动态风险分析基本原理的探 讨, 灾害学, Vol.30, No.2, (2015), 1-7,

[18] 王强. “银行风险雷达图” 在我国商业银行风险 监测中的应用. 上海金融, 2000(5):25-27.

[19] T. Wu, C.F. Huang, F.L. Ai. A discussion on using internet of intelligences to improve risk radar. Intelligent Systems and Decision Making for Risk Analysis and Crisis Response, Eds. C.F. Huang, C. Kahraman. Boca Raton, USA: CRC Press, pp. 831-837, 2013

[20]Hakikur Rahman. Community Based Disaster Information Management System: Perspective Bangladesh. Bangladesh: The Regional Workshop on Best Practices in Disaster Mitigation, 2002.

[21] 吴䑣. 智联网驱动的在线风险雷达在在社区应 急管理中的应用研究. 北京: 北京师范大学, 2015.

[22] 徐宗本,张讲社.基于认知的非结构化信息处理: 现状与趋势.中国基础科学.2007, (06): P53-55.

许学标, 顾宁. 半结构化数据模型及查询语言. 计算 机研究与发展, 1998(10):896-901.

[23] 张德政,张萍萍.非结构化信息管理.微计算机信 息.2006,(09)：P218-219

[24] 万里鹏. 非结构化到结构化数据转换的研究与 实现. 西南交通大学, 2013.

[25] W.D. Wang, S. Qiao, F.L. Zeng, J. Guo. An approach to ranking integrated models for risk assessment by using the internet of intelligences. huang. Journal of Risk Analysis and Crisis Response, 2016, 6(1): 2-9. 